\title{
Determining the Origin of Inner Planetary System Debris Orbiting the Dustiest Main Sequence Stars
}

\author{
Carl Melis ${ }^{1}$, B. Zuckerman ${ }^{2}$, Joseph H. Rhee ${ }^{3}$, Inseok Song ${ }^{4}$, \\ S. J. Murphy ${ }^{5}$, and M. S. Bessell ${ }^{5}$ \\ ${ }^{1}$ Center for Astrophysics and Space Sciences, University of California, San Diego, California \\ 92093-0424, USA; email: cmelis@ucsd.edu \\ ${ }^{2}$ Department of Physics and Astronomy, University of California, Los Angeles, California \\ 90095-1547, USA \\ ${ }^{3}$ Department of Physics and Astronomy, California State Polytechnic University, Pomona, \\ Pomona, California 91768, USA \\ ${ }^{4}$ Department of Physics and Astronomy, University of Georgia, Athens, Georgia 30602, USA \\ ${ }^{5}$ Research School of Astronomy and Astrophysics, College of Mathematical and Physical \\ Sciences, The Australian National University, Cotter Road, Weston Creek, Australian Capital \\ Territory 2611, Australia
}

\begin{abstract}
We develop a means to distinguish between warm dusty circumstellar debris disks produced by steady state collisional evolution of a planetesimal belt or through transient events.
\end{abstract}

Keywords. planets and satellites: formation, circumstellar matter, planetary systems: formation

\section{Introduction}

Dusty circumstellar disks orbiting main sequence stars other than the Sun have been known since the discovery of a dusty shell around Vega by the InfraRed Astronomical Satellite (Aumann et al. 1984). Since then significant advances have been enabled by modern satellites with dedicated debris disk observational programs. A major goal of these recent surveys is to determine how frequent exo-zodiacal dust belts are around other stars and what the origin of their dusty material is.

Searches by our team have uncovered some of the dustiest main sequence stars known (e.g., BD+20 307, Song et al. 2005; EF Cha, Rhee et al. 2007; HD 23514, Rhee et al. 2008; HD 15407, Melis et al. 2010; V488 Per, Zuckerman et al. 2012; TYC 824126521 , Melis et al. 2012; HD 131488 and HD 121191, Melis et al. 2012 ApJ submitted). With these exceptionally dusty systems we seek to probe major formation events for rocky, terrestrial-like planets. To do so we develop an analytical methodology for distinguishing between dust production through collisions of small, numerous rocky bodies in a region akin to the asteroid belt in our Solar system and those produced by giant impacts (previously referred to in Rhee et al. 2008 and Melis et al. 2010 as "catastrophic collisions") between a small number of planetary embryos or planets. Giant impacts are expected to be the final mass accretion events for terrestrial planets and hence herald the existence of massive rocky bodies in a star's terrestrial planet zone (e.g., Stewart \& Leinhardt 2012, and references therein). 


\section{Transient or Steady-state Dust Production?}

To classify systems with terrestrial planet-zone dust into these two regimes - an active planetesimal belt or giant impacts - we begin with the assumption that all inner planetary system dust disks are the product of collisions of numerous small rocky bodies in an active planetesimal belt. We then estimate what total mass of parent bodies is necessary to provide the observable disk parameters and compare this mass to the model simulations of Kenyon \& Bromley (2006) for the formation of terrestrial planets. Should the mass of planetesimals be sufficient to coalesce into one or more rocky planet-sized objects, then we reject the original assumption that the dust is being produced in an active planetesimal belt and instead conclude that the dust was produced in a transient event connected to the formation of a rocky planet. For the systems considered by our team, the copious amounts of dust are strongly suggestive of a collision between two large rocky bodies and hence a giant impact type event.

The analytical method begins with the expression for steady-state mass loss from a massive asteroid belt (Chen \& Jura 2001; Chen et al. 2006):

$$
M_{P B} \gtrsim t_{\text {age }} \times \frac{M_{\text {dust }}}{t_{\text {loss }}}
$$

where $M_{P B}$ is the total mass in parent bodies in the planetesimal belt, $t_{a g e}$ is the age of the stellar system, and $t_{\text {loss }}$ is the timescale of the dominant dust removal mechanism operating in the disk. The value used for $t_{\text {age }}$ typically should be the best measured stellar age available, as in the case of $\zeta$ Leporis $\left(t_{\text {age }}=300 \mathrm{Myr}\right.$; Chen \& Jura 2001). However, in the case of young stars with ages $\lesssim 20 \mathrm{Myr}$, steady state collisions between rocky objects (or even transient collisional events between rocky bodies) has likely been progressing for a period of time notably less than the age of the star. This is realized through considering the gas dispersal timescale for protoplanetary disks (e.g., Zuckerman et al. 1995; Currie et al. 2009) and the assumption that destructive or accretionary planetesimal collisions in inner planetary systems do not begin until after the majority of the primordial disk gas has dispersed. Thus, the value used for $t_{\text {age }}$ must be adjusted for the time in which the gaseous disk component is still dominant. Based on the findings of Zuckerman et al. (1995) and Currie et al. (2009), we take this length of time to be $\sim 5$ Myr.

Chen \& Jura (2001) consider the case of $\zeta$ Leporis and assume that Poynting-Robertson (PR) drag is the dominant dust removal process. We make use of the following equation for the Poynting-Robertson timescale (e.g., Burns et al. 1979):

$$
t_{p r}=\left(\frac{4 \pi\langle a\rangle \rho_{s}}{3}\right) \frac{c^{2} R_{d u s t}^{2}}{L_{*}},
$$

where $R_{\text {dust }}$ is the dust orbital separation from the host star as given below (Equation 2.6), $\rho_{s}$ is the grain density, $L_{*}$ is the stellar luminosity, and $\langle a\rangle$ is given as follows (e.g., Chen et al. 2006):

$$
\langle a\rangle=\frac{5}{3} a_{\min }=\frac{5}{3} \frac{3 L_{*} Q_{p r}}{16 \pi G M_{*} c \rho_{s}} .
$$

Here $a_{\min }$ is the minimum grain size in the disk, the factor of $\frac{5}{3}$ comes from integrating $N(a) d a$ weighting by the number of particles (e.g., Chen \& Jura 2001), and $Q_{p r}$ is the radiation pressure coupling coefficient of the grains and is taken to be $\sim 1$ (Chen et al. 2006).

Following Rhee et al. (2008) and Melis et al. (2010) we also consider a loss mechanism for dust particles due to collisional grinding of debris followed by radiative blowout of 
small particles. The collisional lifetime of dust grains in a given disk as a function of a primary observable - in our case the fractional infrared luminosity $\left(\tau=\mathrm{L}_{I R} / \mathrm{L}_{b o l}\right)$ - is gleaned from various derivations in the literature. Usually collision rates are expressed not as a function of $\tau$ but rather in terms of what has been called "face-on fractional surface density $\sigma(\mathrm{r})$ " (Backman \& Paresce 1993) or "full vertical optical thickness" (Artymowicz \& Clampin 1997) of disk-like structures. Zuckerman \& Song (2012) discuss how to relate $\tau$, an optical depth along the radial direction, to perpendicular (vertical) optical depth. The resulting expression involves $(\Delta \mathrm{R}) / \mathrm{R}$ of the debris disk, where $\Delta \mathrm{R}$ is the disk width in the radial direction. Then with typical $(\Delta \mathrm{R}) / \mathrm{R}=0.3$, as measured for stars with spatially resolved debris disks, collision times may be written as:

$$
t_{\text {coll }}=\frac{P_{\text {orb }}}{80 \times \tau} .
$$

This expression agrees with collision times given in Artymowicz \& Clampin (1997) and Wyatt et al. (2007). The collision time scale can be further expanded into host star (the mass of the star, $M_{*}$, the stellar temperature, $T_{*}$, and the stellar radius, $R_{*}$ ) and dust-specific (the dust temperature, $T_{d u s t}$ ) parameters with the following relations:

$$
\begin{array}{r}
P_{\text {orb }}=\frac{2 \pi}{\sqrt{G M_{*}}} R_{d u s t}^{\frac{3}{2}}, \\
R_{\text {dust }}=\frac{1}{2}\left(\frac{T_{*}}{T_{\text {dust }}}\right)^{2} R_{*} .
\end{array}
$$

Here we have assumed that the dust grains are blackbody-like in nature to relate the dust orbital semi-major axis $\left(R_{d u s t}\right)$ to the dust temperature (Equation 2.6). This assumption of blackbody-like grains is validated by the discussion presented in Rodriguez \& Zuckerman (2012) where it is found that debris disk physical sizes are roughly comparable to (within a factor of 1-5 of) their blackbody-grain emission estimated sizes as long as the physical size is determined with observations near the peak wavelength of disk emission.

The dominant mass loss mechanism is the shorter of the collision timescale and the Poynting-Robertson drag timescale which are related by:

$$
\frac{t_{\text {coll }}}{t_{p r}}=\frac{1}{2640 \times \tau} \frac{T_{d u s t}}{T_{*}} \sqrt{\frac{M_{*}}{R_{*}}}
$$

where $t_{c o l l}$ and $t_{p r}$ are the collision and Poynting-Robertson dust removal timescales respectively, $\tau$ is the fractional infrared luminosity of the dust disk $\left(\mathrm{L}_{I R} / \mathrm{L}_{*}\right), T_{d u s t}$ is the dust disk temperature, $T_{*}$ is the stellar effective temperature, $M_{*}$ is the stellar mass in Solar units, and $R_{*}$ is the stellar radius in Solar units. For the case of $\zeta$ Leporis we find that Equation 2.7 yields $t_{\text {coll }} \approx 0.08 t_{p r}$, indicating that the collision timescale is an order of magnitude faster than the Poynting-Robertson timescale. The above expression, when applied to all systems with detectable mid-infrared excess emission above the stellar photosphere, indicates that collisional dust removal is the dominant grain-loss mechanism (see e.g., the low dust luminosity systems studied by Morales et al. 2009, especially the values given in their Table 3 , but note that they assume $\Delta \mathrm{R} / \mathrm{R}=0.1$ ). This remains true even if $R_{d u s t}$ is larger than the blackbody estimated size by a factor of 1-5 (Rodriguez \& Zuckerman 2012) since $t_{p r}$ increases like $R_{\text {dust }}{ }^{2}$ and $t_{\text {coll }}$ like $R_{\text {dust }}{ }^{3 / 2}$.

When collisions dominate the mass loss, then one can insert $t_{\text {coll }}$ into Equation 2.1 as $t_{\text {loss }}$ and combine it with an expression for the minimum dust mass in a disk system (e.g., Jura et al. 1995): 


$$
M_{\text {dust }} \geqslant \frac{16 \pi}{3} \frac{L_{I R}}{L_{*}} \rho_{s} R_{d u s t}^{2}\langle a\rangle .
$$

Expressing $L_{I R} / L_{*}$ as $\tau$ and exchanging $L_{*}$ in $\langle a\rangle$ for $4 \pi R_{*}^{2} \sigma_{S B} T_{*}^{4}$ one may then arrive at the following:

$$
M_{P B} \gtrsim\left(2.8 \times 10^{20}\right) \frac{t_{\text {age }} \tau^{2}}{T_{\text {dust }}} R_{*}^{2.5} T_{*}^{5} M_{*}^{-0.5}
$$

where $M_{P B}$ is in grams, $t_{a g e}$ is in seconds, $T_{d u s t}$ in $\mathrm{K}$, and $R_{*}, T_{*}$, and $M_{*}$ in Solar units. Since $t_{\text {coll }}$ is used in deriving Equation 2.9, it is assumed that $\Delta R / R=0.3$ (see Equation 2.4 and preceding discussion). The right hand side of Equation 2.9 - when everything except $t_{\text {age }}$ is multiplied together - gives the rate in $\mathrm{g} \mathrm{s}^{-1}$ of the loss of small dust particles due to a collisional cascade followed by radiative blowout.

To place estimated parent body masses into context we turn to the modeling work of Kenyon \& Bromley (2006), in particular their simulation of the growth of terrestrial planets in an annular ring spanning 0.84-1.16 AU around a Solar-mass star. This ring is seeded with a parent planetesimal population having a specified surface density distribution $\Sigma_{0}$. It is then evolved using the hybrid $n$-body-coagulation code as described by Kenyon \& Bromley (2006) and Bromley \& Kenyon (2006). The results of their simulations suggest that for initial surface densities of $\Sigma_{0} \approx 1-12 \mathrm{~g} \mathrm{~cm}^{-2}$ terrestrial planets will form, although for surface densities of $\approx 1.2 \mathrm{~g} \mathrm{~cm}^{-2}$ planets more massive than Mars do not form. This range of surface densities corresponds to a total mass of parent bodies in the annular ring of $\approx 0.1-1 \mathrm{M}_{\text {Earth }}$. To be conservative in our choice of ring masses that should coalesce into planets, we adopt an Earth-mass as a dividing line for distinguishing between active planetesimal belts and colliding planetary embryos. That is, if $M_{P B} \gtrsim 1 \mathrm{M}_{\text {Earth }}$ then we conclude that the inner planetary system material originates from the collision of large rocky bodies and hence that terrestrial planets are forming around the star in question.

One should note that the modeling work of Kenyon \& Bromley (2006) was tailored for Solar-mass stars and to date no similar models exist for stars of intermediate-mass, though we note the work of Kennedy \& Kenyon (2008) where in their analytical approach they derived stellar mass-dependant relations for Super-Earth formation beyond the snow line around A-type stars. Thus, the blanket application of this method to all stellar mass regimes may not be appropriate. Additionally, although the method outlined above is likely sufficient for selecting a statistical sample of stars that are probably undergoing terrestrial planet formation, it should not be viewed as an absolute rubric for the selection of systems that recently endured a giant impact type event.

\section{References}

Artymowicz, P. \& Clampin, M. 1997, ApJ, 490, 863

Aumann, H. H., et al. 1984, ApJL, 278, L23

Backman, D. E. \& Paresce, F. 1993, in Protostars and Planets III, edited by E. H. Levy \& J. I. Lunine, $1253-1304$

Bromley, B. C. \& Kenyon, S. J. 2006, AJ, 131, 2737

Burns, J. A., Lamy, P. L., \& Soter, S. 1979, Icarus, 40, 1

Chen, C. H. \& Jura, M. 2001, ApJL, 560, L171

Chen, C. H., et al. 2006, ApJS, 166, 351

Currie, T., Lada, C. J., Plavchan, P., Robitaille, T. P., Irwin, J., \& Kenyon, S. J. 2009, ApJ, 698,1 
Jura, M., Ghez, A. M., White, R. J., McCarthy, D. W., Smith, R. C., \& Martin, P. G. 1995, $A p J, 445,451$

Kennedy, G. M. \& Kenyon, S. J. 2008, ApJ, 682, 1264

Kenyon, S. J. \& Bromley, B. C. 2006, AJ, 131, 1837

Melis, C., Zuckerman, B., Rhee, J. H., \& Song, I. 2010, ApJL, 717, L57

Melis, C., Zuckerman, B., Rhee, J. H., Song, I., Murphy, S. J., \& Bessell, M. S. 2012, Nature, 487, 74

Morales, F. Y., et al. 2009, ApJ, 699, 1067

Rhee, J. H., Song, I., \& Zuckerman, B. 2007, ApJ, 671, 616

- 2008, ApJ, 675, 777

Rodriguez, D. R. \& Zuckerman, B. 2012, ApJ, 745, 147

Song, I., Zuckerman, B., Weinberger, A. J., \& Becklin, E. E. 2005, Nature, 436, 363

Stewart, S. T. \& Leinhardt, Z. M. 2012, ApJ, 751, 32

Wyatt, M. C., Smith, R., Greaves, J. S., Beichman, C. A., Bryden, G., \& Lisse, C. M. 2007, ApJ, 658, 569

Zuckerman, B., Forveille, T., \& Kastner, J. H. 1995, Nature, 373, 494

Zuckerman, B., Melis, C., Rhee, J. H., Schneider, A., \& Song, I. 2012, ApJ, 752, 58

Zuckerman, B. \& Song, I. 2012, ApJ, 758, 77 